\title{
CLÁSSICOS E NOVOS BIOMARCADORES DE SEPSE NEONATAL
}

\author{
CLASSICAL AND NEW BIOMARKERS OF NEONATAL SEPSIS
}

\author{
ALEXANDRE PAULO MACHADO, REBECA MELO ZURITA, CLAUDIA BONADIMAN DE LIMA, ARIELY TEOTÔNIO BOR- \\ GES, BRUNA LUIZA FERNANDES, FERNANDA BACAGINI GUEDES, MARTA CAROLINA MARQUES SOUSA
}

\section{RESUMO}

Objetivo: analisar quais os principais biomarcadores empregados no diagnóstico da sepse neonatal descritos na literatura.

Métodos: foram consultadas as bases de dados dos sistemas eletrônicos Cochrane Library, PUBMED e SciELO por meio dos seguintes descritores combinados: [Neonatal OR Infants OR Newborns AND Sepsis AND Molecular Markers]. Foram incluídos artigos randomizados publicados entre 1990 a 2014 que tratavam de "marcadores infecciosos e/ou inflamatórios envolvidos na sepse neonatal" e dispunham de versão integral disponível gratuitamente no Portal de Periódicos CAPES. Resultados: do total de 655 artigos, 283 foram incluídos, sendo 47\% sobre inflamação, 37\% abordando inflamação/infecção e 16\% infecção. Destes, 184 estavam disponíveis. Dos marcadores encontrados, os mais citados foram: PCR, PCT, IL-6, IL-10, IL-8, TNF- $a$, CD64, E-selectin, IL-1 $\beta$, nGAL, TBARS e SAA.

Conclusão: o diagnóstico por meio de biomoléculas pode ser influenciado de acordo com as particularidades das amostras, dos indivíduos estudados, características das comorbidades e pelas metodologias empregadas. A utilização conjunta dos marcadores moleculares parece ser uma tendência, cada vez mais, empregada na sepse neonatal. Por isso, esta revisão poderá contribuir em investigações futuras, uma vez que, descreve os marcadores que têm sido relatados para 0 diagnóstico de SIRS e sepse neonatal.

Palavras-Chave: biomarcadores, sepse neonatal, recém-nascidos, neonatos

\section{ABSTRACT}

Objective: to analyze the most used biomarkers in the diagnosis of neonatal sepsis described in literature.

Methods: we searched electronic databases of Cochrane Library, PubMed and SciELO, with the following combined descriptors: [Neonatal OR Newborn OR Infants AND Sepsis AND Molecular Markers]. Randomized articles, from 1990 to 2014 dealing with "infectious and/or inflammatory biomarkers involved in neonatal sepsis" which had full version available for free were included.

Results: from a total of 655 studies, 283 were included, $47 \%$ of inflammation, $37 \%$ of inflammation/ infection, and $16 \%$ of infection. From those, 184 were available, and most cited markers were: PCR, PCT, IL-6, IL-10, IL-8, TNF- $\alpha$, CD64, E-selectin, IL-1ß, nGAL, TBARS and SAA.
Rebeca Melo Zurita Correio

Faculdade de Medicina, Universidade Federal de Mato Grosso, Cursa Medicina na Universidade Federal do Mato Grosso (UFMT).

Claudia Bonadiman de Lima Correio Faculdade de Medicina, Universidade Federal de Mato Grosso, Graduanda em Medicina

\section{Ariely Teotônio Borges Correio}

Faculdade de Medicina, Universidade Federal de Mato Grossol, graduação em FARMÁCIA pela Universidade Estadual de Goiás(2009) e graduada em Medicina pela Universidade Federal de Mato Grosso.

Bruna Luiza Fernandes Correio

Faculdade de Medicina, Universidade Federal de Mato Grosso, Graduanda em Medicina

Fernanda Bacagini Guedes Correio

Faculdade de Medicina, Universidade Federal de Mato Grosso, Graduada em Medicina

Marta Carolina Marques Sousa Correio

Faculdade de Medicina, Universidade Federal de Mato Grosso, Graduada em Medicina 
Conclusion: diagnosis using biomolecules can be influenced by samples particularities, studied subjects, comorbidities particularities and employed assay. The combined use of molecular markers in neonatal sepsis seems to be a trend, increasingly common in the majority of the researches. Therefore, this review may help in future investigations, since it describes the reported markers for diagnosis of SIRS and neonatal sepsis.

KeyWords: biomarkers, neonatal sepsis, newborns, neonates.

Introdução

A definição de sepse foiestabelecida no ano de 1991 na Conferência de Consenso de Sepse, e permanece utilizada até a atualidade. A sepse foi denominada de Síndrome da Resposta Inflamatória Sistêmica (SIRS) e conceituada como decorrente de um processo infeccioso comprovado. Para que a SIRS seja diagnosticada é necessária a presença de pelo menos dois dos seguintes sintomas: febre ou hipotermia; taquicardia; taquipneia; e leucocitose ou leucopenia.1

Os recém-nascidos ( 0 a 7 dias de vida) e neonatos (7 dias a 1 mês), em especial os prematuros de baixo peso com mães que possuem infecções, e que sofreram ruptura prematura de membrana amniótica, podem ser acometidos pela sepse neonatal (SN), que é classificada como precoce quando ocorre nos primeiros sete dias de vida e é causada por microrganismos adquiridos durante o parto, e tardia após esse período, com etiologia nosocomial ou comunitária. 0 aparecimento da sepse no recém-nascido (RN) ou neonato, em geral é insidioso, possui sinais e sintomas inespecíficos, particularmente no pré-termo, e está associado com os procedimentos invasivos somados à imaturidade imunológica. Em decorrência desses fatores a sepse neonatal continua sendo uma patologia de elevada morbimortalidade, principalmente nos países em desenvolvimento.2-7

A hemocultura é considerada o padrão-ouro no diagnóstico da sepse neonatal. No entanto, sua positividade é variável e os resultados não são disponibilizados rapidamente para definição de uma conduta terapêutica adequada. Por esse motivo, proteínas de fase aguda como a proteína C-reativa (PCR) e a procalcitonina (PCT), são empregados para o diagnóstico precoce da sepse neonatal. Outros marcadores moleculares ainda são descritos, como as interleucinas (IL-1, LL-6, IL-8 e IL-10), fator de necrose tumoral (TNF), seus receptores solúveis (receptor do TNF) e fatores estimuladores de colônias (G-CSF, M-CSF, GM-CSF). Recentemente mais marcadores estão sendo pesquisados, principalmente a amiloide sérica $\mathrm{A}(\mathrm{SAA})$, o receptor $\mathrm{CD64}$ de neutrófilo, as substâncias reativas ao ácido tiobarbitúrico e a interleucina $\beta$ $(\mathrm{IL}-\beta) .4$

Todos esses marcadores biológicos, quando associados à avaliação clínica, aumentam as chances de acerto diagnóstico e trazem mais segurança para o início precoce da administração de antimicrobianos e melhor prognóstico dos neonatos. Dessa forma, o objetivo deste trabalho foi conhecer os principais marcadores para a SIRS e sepse, clássicos ou novos, e estabelecer suas validades enquanto substâncias capazes de diagnosticar com rapidez e eficiência a sepse neonatal.

\section{Metodologia}

Este estudo consistiu em uma revisão literatura sobre os biomarcadores mais empregados em métodos de diagnóstico molecular da sepse neonatal.

Estratégia da Pesquisa

Foram utilizadas referências de três bases de dados eletrônicos (Cochrane Library, PUBMED e SciELO) com os seguintes descritores combinados: [Neonatal OR Infants OR Newborns AND Sepsis AND Molecular Markers]. Estudos randomizados publicados entre os anos de 1990 a 2014 foram incluídos. Foram incluídos somente artigos originais e comunicações com dados laboratoriais. $O$ banco de dados foi construído entre os meses de junho a novembro de 2014 Os artigos selecionados foram separados por assunto: infecção, inflamação e infecção/inflamação; sendo cada tema distribuído para dois revisores cegos e independentes. A inclusão dos trabalhos se baseou na leitura de títulos e resumos, observando se os mesmos tratavam de "marcadores infecciosos, inflamatórios, ou ambos envolvidos na sepse neonatal". As publicações que apresentavam versões disponibilizadas na íntegra através do Portal de Periódicos CAPES/MEC ou com acesso gratuito foram incluídas. Para tabulação dos dados, os artigos foram classificados de acordo como três idiomas principais: português, inglês e espanhol. Em seguida, as tarefas foram divididas entre os revisores com a finalidade de reunir os dados coletados em planilhas para análise quanti/qualitativa. Foram obtidas fotocópias completas dos artigos selecionados para posterior avaliação em grupo.

Resultados

Os artigos foram pesquisados em três bases de dados: PubMed (A1), Scielo (A2) e Cochrane (A3), conforme fluxograma 1. Um total de 655 artigos foram selecionados, resultando em $283(A 1=267, A 2=2$ e A3=14) incluídos, dos quais 184 $(A 1=174, A 2=2$ e $A 3=8)$ estavam disponiveis e 98 ( $A 1=95, A 2=0$ e $A 3=3$ ) indisponiveis. Excluiu-se por título 297 artigos $(A 1=282, A 2=5$ e $A 3=10)$ e por resumo 89 artigos $(A 1=89, A 2=0$ e $A 3=0)$. Utilizaram-se somente os artigos incluídos da base Pubmed porque foram encontradados dados similares nas bases Scielo e Cochrane.

Os artigos selecionados foram publicados entre 1990 e 2014. Dos 283 artigos incluídos, houve maior número de publicações sobre o tema nos anos de 2004, 2007, 2008, 2010, 2012 e 2013. A partir de 1997 houve aumento no número de publicações na área do conhecimento estudada, conforme gráfico 1.

A partir da análise dos 283 artigos incluídos, como pode ser visto no Gráfico 2, constatou-se que $47 \%$ abordavam majoritariamente marcadores de inflamação. Enquanto que, $37 \%$ dos artigos restantes tratavam de marcadores de inflamação e infecção e 16\% de infecção.

Discussão

O diagnóstico de sepse tem sido sugerido de acordo com as manifestações clínicas SIRS e testes laboratoriais de rotina, podendo ser posteriormente confirmado pelo isolamento do agente etiológico. No entanto, nem sempre é possível estabelecer a confirmação precoce do quadro séptico com base somente em tais critérios diagnósticos, o que pode implicar num mau prognóstico devido ao início tardio das intervenções critérios diagnósticos, o que pode implicar num mau prognóstico devido ao início tardio das intervençōes
terapêuticas. A utilização de biomarcadores moleculares tem se mostrado promissora na obtenção de resultados presuntivos rápidos e de alta acurácia. E, embora exista a necessidade de equipamentos especiais e profissionais qualificados, o emprego de biomoléculas acaba sendo mais vantajoso em relação ao custo-benefício quando comparado a métodos tradicionais. 8,9

Nos últimos anos, um grande número de marcadores para diagnóstico de sepse neonatal tem sido investigado. Neste sentido, a presente revisão, teve por objetivo verificar quais os biomarcadores foram descritos na sepse neonatal de 1990 a 2014. Assim, no decorrer da análise (Fluxograma 1), notou-se que variantes particulares em cada artigo quanto à amostra (sexo, idade, comorbidade analisada) e aos biomarcadores (análise isolada ou combinada entre biomoléculas) influenciaram os resultados de sensibilidade, especificidade, valor preditivo positivo (VPP) e negativo (VPN).

Alguns biomarcadores, tais como PCR, PCT, CD64, SAA, E-selectina, nGAL, TBARS e algumas citocinas, têm sido convencionalmente úteis nos testes laboratoriais e pesquisas envolvendo suspeita de sepse, os quais vêm sendo validados como moléculas que auxiliam não somente no diagnóstico, mas também na classificação da gravidade e prognóstico da doença.8

Marcadores mais convencionalmente utilizados

A proteína PCR vem sendo utilizada, nos últimos 10 anos, como um marcador inflamatório de fase aguda em exames de rotina. Após acometimento por processo infeccioso ou lesão tecidual, a síntese de PCR se inicia de 6 a 8 horas.10 Atinge pico de concentração sérica depois de 2 a 3 dias e, caso a evolução do quadro seja favorável, segue-se em decréscimo, tendo tempo de meia vida aproximadamente de 19 horas. A sensibilidade deste marcador aumenta-se consideravelmente de 24 e 48 horas após o estabelecimento da sepse.10,4 Diante deste contexto, a PCR vem sendo considerada como um marcador tardio e específico de infecção, porém quando é analisada isoladamente no sangue umbilical tem revelado pouca utilidade 
para detecção de sepse neonatal de início precoce. No pós-natal, a concentração pode aumentar em até 1000 vezes no recém-nascido durante uma resposta de fase aguda. A especificidade da PCR pode ser acessível na sepse de aparecimento precoce. No entanto, na ausência de infecção sistêmica, as ocorrências falso-positivas têm sido relacionadas, em especial, a certas condições pré-natais, tais como febre materna, sofrimento fetal ou parto fatigante. A PCR também vem sendo bastante útil no manejo das infecções nosocomiais tardias, incluindo enterocolite necrotizante.

A procalcitonina (PCT), pró-hormônio da calcitonina, ocorre em concentrações muito baixas em indivíduos saudáveis e seus níveis aumentam significativamente na sepse bacteriana (especialmente na severa e choque séptico). Normalmente, sua liberação ocorre 3 horas após a presença de endotoxinas. Os níveis séricos de PCT também aumentam em outras condições como: cesariana, ruptura de membrana $<18 \mathrm{~h}$, corioamnionite, ressuscitação ao nascimento, exposição pré-natal a corticoesteróides e doenças respiratórias não-infecciosas (em exemplo, doença da membrana hialina e síndrome do desconforto respiratório), o que pode dificultar a interpretação de seus níveis.11,12 A dosagem da PCT pode ser usada para discriminar as inflamações sistêmicas da sepse bacteriana, além de ser útil para monitorar o progresso e avaliar o prognóstico do paciente.13 Também se concluiu a partir de tal estudo que, quando usada como único marcador, a PCT não é suficientemente confiável.

Em análise prospectiva multicêntrica verificou-se que a confiabilidade da PCT como marcador reque valores específicos de corte para cada momento de avaliação $(0,55 \mathrm{ng} / \mathrm{ml}$ ao nascimento; $4,7 \mathrm{ng} / \mathrm{ml}$ de 12 às $24 \mathrm{~h} ; \mathrm{e} 1,7 \mathrm{ng} / \mathrm{ml}$ de 36 às $48 \mathrm{~h}$ ). Porém, há falta de precisão sobre os valores normais a serem usados como parâmetros na avaliação dos níveis da PCT.14

Em um estudo multicêntrico realizado com recém-nascidos pré-termo e a termo com suspeita de sepse nos primeiros três dias de vida, demonstrou-se que a procalcitonina pode ser utilizada como marcador para encurtar a duração da antibioticoterapia nos casos de sepse neonatal precoce.15

Análise combinada entre marcadores clássicos e modernos

Khassawneh et al. compararam a utilização da IL-6 e da PCR para o diagnóstico de sepse neonatal precoce.16 O melhor parâmetro encontrado foi de PCR com $95 \%$ de sensibilidade e $98 \%$ de valor preditivo negativo Valores ótimos de corte para (IL-6, TNFa, PCR, E-selectina e a IL-1ß) foram definidos para detecção de infecção tardia sistêmica em prematuros de muito baixo peso ao nascer. Demonstrou-se que a avaliação concomitante dos níveis de lL-6 ePCRouTNF-a pode contribuir com diagnóstico da sepsee descontinuidade do tratamento após 48 horas, sem esperar pelos resultados microbiológicos, desde que os bebês estejam em boas condições clínicas. 17

A combinação de dois marcadores IL-6 e PCR parece ser mais adequada para o diagnóstico precoce no dia 0 e até 24 horas de evolução do quadro, com sensibilidade e valor preditivo negativo de $100 \%$. $O$ diagnóstico de sepse empregando IL6 e PCR e o resultado dos testes, no decorrer dos dias, acompanhou a evolução clínica dos neonatos. 18

Edgar et al. realizaram estudo prospectivo para avaliação da PCR altamente sensível, molécula de adesão intercelular 1 solúvel (ICAM-1), E-selectina solúvel e SAA como marcadores de sepse neonatal. Foi sugerido que os quatro marcadores têm valor diagnóstico para sepse e quando utilizados conjuntamente apresentam um alto valor preditivo negativo. 19

Beceiro-Mosquera et al. realizaram estudo comparando IL-6 e PCR em 42 recém-nascidos com suspeita de infecção clínica. IL-6 e PCR foram considerados bons marcadores de infecção quando o valor de IL-6 fo maior que $96 \mathrm{pg} / \mathrm{mL}$, ou quando houve combinação de IL-6 maior que $53 \mathrm{pg} / \mathrm{mL}$ e PCR maior que 13,3mg/L, tendo atingido $100 \%$ de especificidade e VPP. Com os resultados obtidos desenvolveram um algoritmo de decisão para o início da antibioticoterapia nos recém-nascidos.20

Os marcadores IL-6, IL-8, PCR e PCT são sensíveis quando há lipopolissacarídeos de membrana de bactérias no início da infecção. Quando comparados os valores de IL-6, IL-8 e PCT aos de PCR no diagnóstico e tratamento da sepse tardia em crianças admitidas na Unidade de Terapia Intensiva Neonatal, concluiu-se que IL-6, IL-8 e PCT são parâmetros melhores que a PCR no diagnóstico e no acompanhamento da sepse neonatal provocada por estafilococos coagulase negativos, inclusive na exclusão de infecção bacteriana entre os internados por infecção enteroviral.21 O diagnóstico da sepse é difícil em recém-nascidos e neonatos internados na UTI devido a sinais clínicos inespecíficos. Portanto, indicadores confiáveis de sepse e seu emprego combinado seriam úteis para um diagnóstico preciso, resultando em redução do uso desnecessário de antibióticos.

O uso da PCT, da PCR e da leucometria revelou que a contagem de leucócitos na admissão do recémnascido não tem valor na diferenciação do tipo de infecção nas crianças criticamente doentes e que a PCT cordão umbilical de 197 recém-nascidos, sendo detectadas altas taxas de sensibilidade e especificidade para PCT.23

Os parâmetros microbiológicos, hematológicos e o marcador SE-Selectina foram investigados como preditores precoces para avaliar o desfecho da sepse neonatal, em uma amostragem com 90 casos (sendo 58 infectados e 32 não infectados) e 30 controles.24 SE-Selectina analisada isoladamente demonstrou sensibilidade de $59 \%$, especificidade de $87 \%$, valor preditivo positivo de $81 \%$ e valor preditivo negativo de $69 \%$. Além de seu valor diagnóstico, principalmente em associação com a PCR, esse marcador pode ser usado como um indicador de prognóstico de septicemia neonatal e pode prever a etiologia a ser apontada na hemocultura (análise combinada). No entanto, ao ser analisada em conjunto com a PCR, houve alteração de tais resultados: $45 \%$ sensibilidade, $100 \%$ especificidade, $100 \%$ valor preditivo positivo e $65 \%$ valor preditivo negativo. Além de que, em análises do subgrupo de casos infectados $(n=58)$, a SESelectina alterou-se de acordo com a etiologia de base. Nos casos de sepse neonatal por bactéria Gramnegativa $(n=42 ; 72 \%)$ houve maiores níveis de SE-Selectina do que em bebês infectados por bactérias Gram-positivas ( $n=16 ; 28 \%$ ); e os níveis plasmáticos de SE-Selectina foram maiores em não sobreviventes e naqueles com disfunção hemodinâmica.

Novos biomarcadores

O soro amiloide A ou Amilóide sérica A (SAA) tem sido usado como novo marcador de inflamação aguda e crônica. SAA é proteína de fase aguda induzida pelas citocinas IL-1 e IL-6 e TNF, em resposta a LPS de bactérias Gram-negativas. Há grande aumento dos níveis de SAA de 8 a 24 horas após o início da sepse. Em estudo envolvendo 104 neonatos, com menos de $72 \mathrm{~h}$ de vida, foi demonstrado SAA como um marcador inflamatório de sepse neonatal precoce.25 Uma maior sensibilidade e níveis mais elevados de SAA ocorriam no início da sepse, tendo retorno da concentração para valores normais durante a recuperação.

O receptor CD64 é expresso em macrófagos, monócitos, neutrófilos e eosinófilos. CD64 é um marcador útil no diagnóstico de infecções tardias. CD64 de neutrófilos tem sido descrito como marcador altamente sensível para sepse neonatal.26,27 CD64 de neutrófilos combinado com outros marcadores e contagem de leucócitos pode ampliar consideravelmente a detecção da condição septicêmica.28

Crianças com sinais de infecção e diagnóstico presuntivo de sepse apresentam níveis significativamente mais altos de Fator de Necrose Tumoral-a (TNF- $a$ ) em relação às outras hígidas.29,30 Os níveis desse marcador foram significativamente mais elevados em crianças mais velhas e em infecções de etiologia por bactérias Gram-negativas. Sua liberação, em geral, ocorre em 30 minutos com pico de concentração em cerca de 1,5 horas. Os níveis de TNF-a decrescem com o avanço da idade gestacional, no entanto alguns resultados contraditórios não demonstram essa diferença em prematuros saudáveis. A concentração inicial nos RN também não é afetada pelo tipo de parto, nem pelo nível sérico maternal, sugerindo que o TNF-a provavelmente não atravesse a placenta durante o parto.

Segundo Hodge et al., o aumento das concentrações séricas de uma citocina em recém-nascidos e neonatos não é suficiente para o estabelecimento de sepse neonatal, todavia, a expressão ou elevação de múltiplas moléculas podem identificar quadro séptico.31 IL-10 foi a citocina que mais se correlacionava quando avaliada isoladamente, encontrada em níveis aumentados em mais de $50 \%$ dos casos. Porém, essa interleucina foi detectada mais tardiamente, quando comparada a IL-6. Esse estudo também concluiu que houve diferenças entre os grupos de idade ( $1^{\mathrm{a}}$, $2^{\mathrm{a}}$ e $3^{\mathrm{a}}$ semanas de vida). IL-10, como também IL-6 e IL-12, teve elevação nos primeiros 7 dias, sugerindo que as infecções intrauterinas podem estar associadas com níveis mais elevados dessas citocinas em tal período, comparando às infecções adquiridas após o nascimento. Boskabadi et al. avaliaram IL-6, IL-8 e IL-10, verificando que ambas são bons marcadores 
para sepse precoce, porém IL-6 demonstrou melhor sensibilidade e correlação com mortalidade acima de $78.2 \mathrm{pg} / \mathrm{mL} .32$

Mães com bebês pré-termos (<28semanas) demonstraram altas concentrações de proteínas protetoras no colostro, tais como: lisozima, HBD1, sCD14 e TGF- $\beta 2$, comparado a mães de neonatos nascidos a termo. 33 Tais fatores maternos, inclusive citocinas, contribuem com a proteção do recém-nascido no período prénatal e pós-natal. No entanto, o uso de corticoides pode influenciar no perfil e taxa desses componentes imunológicos no pós-natal. Portanto, utilizar as citocinas pró ou anti-inflamatórias na sepse neonatal como único parâmetro têm os seus riscos. A detecção de múltiplas citocinas tem sido mais empregada, particularmente de L-6, IL-8, IL-10 e TNF- $a$, as quais têm sido detectadas nas infecções de crianças34 e adultos. Os marcadores IFN- $\gamma$, IL-10, IL-18, TGF- $\beta$ e TNF- $\alpha$, mensurados durante as três primeiras semanas de vida, foram associados significativamente com sepse em lactentes de muito baixo peso.35

O perfil de marcadores proteômicos de inflamação intra-aminiótica (defensinas de neutófilos humanos 1 e 2 e calgranulinas $A$ e C) foi usado para estabelecer uma pontuação de massa restrita (MR), essa pontuação foi relacionada com a presença de inflamação materna.36 MR de 3 a 4, correspondente a graus avançado de funisite, tiveram relevância como marcador de sepse precoce. Mães com MR maior que 3-4 foram relacionadas a neonatos de menores idades gestacionais, menor peso ao nascimento e maior incidência de sepse (suspeita e confirmada), em comparação aos recém-nascido de mães com MR menores do que 1 ou 2.

De acordo com o levantamento bibliográfico, observa-se também outros novos biomarcadores para sepse neonatal, os quais vem sendo utilizados nas pesquisas com frequência (citados de 2 a 5 vezes), são eles: TBARS (substâncias reativas aos ácidos tiobarbitúricos), G-CSF (fator estimulador de granulócitos), UNGAL e as proteínas carboniladas. Além destes, apareceram como novos biomarcadores duas vezes citados em pesquisas: a resistina, Pro-ADM (pró-adrenomedulina), lactato, IP-10 (interferon- $\gamma$ induzido por proteína-10), as interleucinas IL-18, IL-12 e IL-2r, Interferon- $\gamma$, cistatina C, neopterin, e LBP (proteína ligada a lipoproteína). No entanto, mais estudos para avaliar estes marcadores e, cada vez mais, novas moléculas estão sendo descritas, o que provavelmente no futuro terá um impacto muito positivo na contenção da SIRS e sepse neonatal.

O diagnóstico de sepse ainda é difícil devido aos sinais clínicos, na maioria das vezes, serem inespecíficos, especialmente em recém-nascidos e neonatos. Portanto, estudos sobre marcadores para diagnóstico da sepse são extremamente relevantes na atualidade. O desenvolvimento de novas tecnologias nesse campo tem aumentado vertiginosamente, o que poderá contribuir com a redução do uso desnecessário de antibióticos e da mortalidade de RN por sepse. Conforme esta revisão, encontramos inúmeros estudos sobre biomarcadores e uma ampla variedade de novas moléculas sendo investigadas. De modo geral observa-se uma aposta mais acentuada ao emprego de múltiplos marcadores moleculares e/ou escores de prognósticos, inclusive no conjunto daqueles que tendem a presunção etiológica, gravidade da doença e apresentam maior sensibilidade e especificidade. A direção desses estudos nesse século aponta que em breve teremos desfechos mais precisos na sepse neonatal.

\section{AGRADECIMENTOS}

Ao Programa de Educação Tutorial do Ministério de Educação pelo apoio financeiro referente ao custeio das bolsas de estudo.
Referencias Bibliográficas

1 Matos GFJ, Victorino JA. Critérios para o diagnóstico de Sepse, Sepse Grave e Choque Séptico. Rev Bras Ter Intensiva. 2004; 16(2): 102-104.

2 Russell AR, Berdford R. Neonatal sepsis. Paediatrics and Child Health. 2011; 21(6): 265-269. 3 Ganatra HA, Stoll BJ, Zaidi AK. International perspective on early-onset neonatal sepsis. Clin Perinatol. 2010; 37(2): 501-23.

4 Caldas JPS, Marba STM, Blotta MHSL, Calil R, Morais SS, Oliveira RTD. Acurácia diagnóstica do leucograma, proteína C-reativa, interleucina- 6 e fator de necrose tumoral-alfa na sepse neonatal tardia. J Pediatr. 2008; 84(6): 536-542

5 Valerio TA, Cancelier AC, Constantino L, Petronilho F, Ritter C, Dal-Pizzol F. Marcadores inflamatórios e oxidativos em sangue de cordão umbilical como preditores de gravidade em sepse neonatal. Rev Bras Ter Intensiva. 2012; 24 (1): 30-34

6 Ceccon, MEJR. Novas Perspectivas na Sepse Neonatal. Pediatria. 2008; 30(4):198-202.

7 Goulart AP, Valle CF, Dal-Pizzol F, Cancelier ACL. Fatores de Risco para o desenvolvimento de sepse neonatal precoce em hospital da rede pública do Brasil. Rev Bras Ter Intensiva. 2006; 18(2): 148-153

8 Siqueira BR, Gomes AP, Calixto LL, Vitorino RR, Perez MCA, Mendonça EG, et al. Sepse: atualidades e perspectivas. Rev Bras Ter Intensiva. 2011; 23(2): 207-216

9 Liu X, Ren H, Peng D. Sepsis biomarkers: an omics perspective. Front Med. 2014; 8(1): 5867.

10 Ng P. Diagnostic markers of infection in neonates. Arch Dis Child Fetal Neonatal Ed. 2004; 89(3): F229-F235.

11 Lee H. Procalcitonin as a biomarker of infectious diseases. Korean J Intern Med. 2013; 28(3):285-91.

12 Wacker C, Prkno A, Brunkhorst FM, Schlattmann P. Procalcitonin as a diagnostic marker for sepsis: a systematic review and meta-analysis. Lancet Infect Dis. 2013; 13(5):426-35.

13 Ballot DE, Perovic O, Galpin J, Cooper PA. Serum procalcitonin as an earlymarker of neonatal sepsis. S Afr Med J. 2004 Oct;94(10):851-4.

14 López Sastre JB, Solís DP, Serradilla VR, Colomer BF, Cotallo GD; Grupo de Hospitales Castrillo. Evaluation of procalcitonin for diagnosis of neonatal sepsis of vertical transmission. BMC Pediatr. 2007; 7:9.

15 Stocker M, HopWC, Van RAM. Neonatal Procalcitonin Intervention Study (NeoPInS): Effect of Procalcitonin-guided decision making on duration of antibiotic therapy in suspected neonatal early-onset sepsis: A multi-centre randomized superiority and non-inferiority Intervention Study. BMC Pediatr. 2010; 10:89.

16 Khassawneh M, Hayajneh WA, Kofahi H, Khader Y, Amarin Z, Daoud A. Diagnostic markers for neonatal sepsis: comparing C-reactive protein, interleukin-6 and immunoglobulin. Scand J Immunol. 2007; 65(2):171-175

17 Ng PC, Cheng SH, Chui KM, Fok TF, Wong MY, Wong W, et al. Diagnosis of late onset neonatal sepsis with cytokines, adhesion molecule, and C-reactive protein in preterm very low birthweight infants. Arch Dis Child Fetal Neonatal Ed. 1997; 77(3):F221-F227.

18 Ceccon ME, Vaz FA, Diniz EM, Okay TS. Interleukins 6 and C-reactive protein for the diagnosis of late onset sepsis in the newborn infant. Rev Assoc Med Bras. 2006; 52(2): 79-85. 
19 Edgar JDM, GabrielV, Gallimore JR, McMillan SA, Grant J. A prospective study of the sensitivity, specificity and diagnostic performance of soluble intercellular adhesion molecule 1, highly sensitive C-reactive protein, soluble E-selectin and serum amyloid A in the diagnosis of neonatal infection. BMC Pediatr. 2010; 10:22.

20 Beceiro Mosquera J, Sivera Monzo CL, Oria de Rueda Salguero O, Olivas López de Soria C, Herbozo Nory C. Usefulness of a rapid serum interleukin- 6 test combined with C-reactive protein to predict sepsis in newborns with suspicion of infection. An Pediatr (Barc). 2009; 71(6): 483-8.

21 Verboon-Maciolek MA, Thijsen SF, Hemels MA, Menses M, van Loon AM, Krediet TG, et al. Inflammatory mediators for the diagnosis and treatment of sepsis in early infancy. Pediatr Res. 2006; 59(3):457-61.

22 Hatherill M, Tibby SM, Sykes K, Turner C, Murdoch IA. Diagnostic markers of infection: comparison of procalcitonin with $C$ reactive protein and leucocyte count. Arch Dis Child. 1999; 81(5): 417-421

23 Joram N, Boscher C, Denizot S, Loubersac V, Winer N, Roze JC, et al. Umbilical cord blood procalcitonin and $C$ reactive protein concentrations as markers for early diagnosis of very early onset neonatal infection. Arch Dis Child Fetal Neonatal Ed. 2006; 91(1):65-66

24 Zaki Mel-S, el-Sayed $\mathrm{H}$. Evaluation of microbiologic and hematologic parameters and E-selectin as early predictors for outcome of neonatal sepsis. Arch Pathol Lab Med. 2009;133(8):1291-6.

25 Arnon S, Litmanovitz I, Regev RH, Bauer S, Shainkin-Kestenbaum R, Dolfin T. Serum amyloid A: an early and accurate marker of neonatal early-onset sepsis. J Perinatol. 2007; 27(5):297-302.

26 Groselj-Grenc M, Ihan A, Derganc M. Neutrophil and monocyte CD64 and CD163 expression in critically ill neonates and children with sepsis: comparison of fluorescence intensities and calculated indexes. Mediators Inflamm. 2008; 2008:202646.

27 Lynema S, Marmer D, Hall ES, Meinzen-Derr J, Kingma PS. Neutrophil CD64 as a diagnostic marker of sepsis: impact on neonatal care. Am J Perinatol. 2015; 32(4):331-6.

28 Yang AP, Liu J, Yue LH, Wang HQ, Yang WJ, Yang GH. Neutrophil CD64 combined with PCT, CRP and WBC improves the sensitivity for the early diagnosis of neonatal sepsis. Clin Chem Lab Med. 2015.

29 Makhija P, Yadav S, Thakur A. Tumor necrosis factor alpha and interleukin-6 in infants with sepsis. Indian Pediatrics. 2005; 42:1024-1028.

30 Kumar S, Rizvi M. Prognostic serum tumor necrosis factor-a in paediatric patients with sepsis. J Infect Dev Ctries. 2009; 3(6): 437-441.

31 Hodge G, Hodge S, Haslam R, McPhee A, Sepulveda H, Morgan E, et al. Rapid simultaneous measurement of multiple cytokines using $100 \mu \mathrm{l}$ sample volumes - association with neonatal sepsis. Clin Exp Immunol. 2004; 137(2): 402-407.

32 Boskabadi $\mathrm{H}$, Maamouri G, Tavakol Afshari J, et al. Evaluation of serum interleukins- 6,8 and 10 levels as diagnostic markers of neonatal infection and possibility of mortality. Iran J Basic Med Sci. 2013; 16(12): $\underline{1232-1237 .}$

33 Trend S, Strunk T, Lloyd ML, Kok CH, Metcalfe J, Geddes DT, et al. Levels of innate immune factors in preterm and term mothers' breast milk during the 1st month postpartum. Br J Nutr. 2016; 115(7): 1178-93. 34Delanghe JR, Speeckaert MM. Translational research and biomarkers in neonatal sepsis. Clin Chim Acta. 2015; 451(Pt A): 46-64.

35 Sood BG, Shankaran S, Schelonka RL, Saha S, Benjamin DK Jr, Sánchez PJ, et al. National Institute of Child Health and Human Development Neonatal Research Network. Cytokine profiles of preterm neonates with fungal and bacterial sepsis. Pediatr Res. 2012; 72(2): 212-20.

36Buhimschi CS, Buhimschi IA, Abdel-Razeq S, Rosenberg VA, Thung SF, Zhao G, et al. Proteomic biomarkers of intra-amniotic inflammation: relationship with funisitis and early-onset sepsis in the premature neonate. Pediatr Res. 2007; 61(3): 318-324. 